\title{
Qualitative and Quantitative Assessment of Patterns of Macular Edema in Diabetic Patients Using Optical Coherence Tomography
}

E.E.Saber, A.A.Fayed, M.G.Sharawy, and A.T.Abo Bakr

Ophthalmology, Dept., Faculty of Medicine, Benha Univ., Benha, Egypt

E-mail: Amaal@yahoo.com

\begin{abstract}
Background: Even in advanced nations, diabetic retinopathy (DR) and Diabetic Macular Edema (DME) are regarded the major reasons for blindness, reducing the quality of life in patients. OCT is a scan method for the whole macular region, which not only measures macular thickness, but also reveals retinal structural alterations. For precise determination of macular thickness, OCT is regarded the most sensitive parameter. The goal of this research was to detect, analyse and link the presently known tomographical patterns of diabetic macular edoema with other parameters such as: BCVA, diabetes duration and $\mathrm{HbA} 1 \mathrm{c}$. Methods: 117 eyes out of 66 patients underwent a study; all patinte were exposed to a visual acuity, a previous sector exam and complete fundus examination. OCT was done to all patients involved. There was no statistically significant relationship with BCVA in the laboratory data. Results and conclusion: In our research, the BCVA\&CMT association is statistically significant. In addition, we discovered central macular edoema $(>300 \mu \mathrm{m})$ as the most significant OCT patterns influencing vision. No significant differences between CMT and HbAlc \& RBS were statistically identified.
\end{abstract}

Keywords: Macular, Edema, DM, Optical, Coherence, Tomography.

\section{Introduction}

A primary cause of visual impairment in diabetic retinopathy is macular oedema. It contains a broad range of pathological abnormalities, including diffuse or localised edoema and cystoid macular edoema with a hard exudate potential. Macular thickness measurement has historically been used to determine the requirement for anti-VEGF or corticosteroid reinjections for disorders such as diabetic macular edoema, retinal retinal edoema and agerelated choroidal neovascularisation. The DRCR.net algorithm for the treatment of diabetic macular oedema is used to indicate retreatment with macular thickness changes. [1]

DME may develop at any stage of DR, however it happens more commonly as diabetes duration and DR severity increases. The ten-year incidence of acquiring DME in patients who had type I diabetes in the Wisconsin Epidemiologic Study of Diabetic Retinopathy (WESDR) was 20.1\%, $13.9 \%$ for patients with type II diabetes who have no insulin and $25.4 \%$ for patients with type II diabetes who use insulin. [2].

DME prevalence rises with DR severity: affects $3 \%$ of the eyes with mild non-proliferative diabetic retinopathy (NPDR), climbs to $38 \%$ of the eyes with moderate to severity of NPDR, reaching $71 \%$ of the eyes of PDR [3].

Normal macular thickness values are normally believed to be the mean and two variances for the machine and sex, which means that normal macular thickness may vary substantially. The thickness of the retina is a pretty weak retinal substitute. For example, if extracellular fluid is in the retina, a retina with neuronal loss and/or glial cells might have a normal thickness. Similarly, disruption to the anatomical retinal connections, for instance eyes disorganizing the inner retinal layers leading to a decreased retinal function might have a normal thickness. The position of the fluid is also important. [4] Prior to the use of the anti-VEGF, a focused laser was the best diabetic macular edoema treatments and led to modest improvement in retinal thinning visual acuity. With this procedure, however, external retinal elements and epithelium of the pigments may be damaged and the retina may be diluted and visual potential may be lost. Ischemic retina, but not infarct, may have normal thickness or may even be expanded, but may not operate properly. Improved imagery of intraretinal capillary systems at all levels may assist track the complete range of neurosensor ischemia, and retinal neuropathic changes may be seen in people with diabetes prior to microvascular modifications, which means the central retina is thicker before the leakage from the capillaries. [5] Several optical instruments were invented for objective evaluation of DME, such the Retina Map, the computerised stereophotographic analyses and the Heidelberg Retina Tomograph. [6] The retinal thickness measured, however the intraretinal structures were not demonstrated. The laser slit implanted in the retina map identifies the intraretinal cyst but does not provide cross-sectional retinal pictures. Therefore several objective approaches have been developed for the quantitative and qualitative evaluation of the retinal edoema, such as optical coherence tomography, based on low-coherence interferometry, which produces high-resolution crosssectional pictures of the retina. It gives data on the internal anatomy of the retinal system and on the quantitative assessment of macular retinal thickness in diabetes patients [7].

The imaging of many disorders affecting the macula, including central serous retinopathy, epiretinal membranes, macular holes, and cystoid macular edoema, has shown effective optical coherence tomography (CME). Four forms of structural alterations have been documented in 
diabetic macular edoema: sponge-like retinal swelling, cystoids macular edoema, serious retinal detachment and traction retinal detachment due to posterior hyaloidal tension. [8]. [8].

The research aims to discover and analyse the presently known pattern of diabetic macular edoema for optical coherence tomography and link this pattern to other factors, such as visual acuity, diabetes time and disease management.

\section{Patients and methods}

Design of the study: prospective observational study.

Study population: diabetic patients admitted to Benha Univesity Hospital with diabetic macular edema.

\subsection{Inclusion criteria}

- Age:25_50 years

- Diabetic for more than ten years

- Eyes with central $1 \mathrm{~mm}$ subfield thickness more than $250 \mu \mathrm{m}$

\subsection{Exclusion criteria}

- Patients who had any treatment for macular edema such as PRP or intravitreal injections.

- Patients with other diseases affect retina such as vascular diseases.

- Patients with retinal dystrophy.

Sample size: one hundred and seventeen eyes who fit the inclusion criteria.

Study tool: 3DTOPCON Optical Coherence Tomography (manufactured by TOPCON TOKYO 2000)

2.3. Included Patients will be subjected to history taking, clinical examination, and full ophthalmologic examination including

- Vision and best corrected visual acuity (BCVA)
- Systemic review: lipid profile, hypertension, blood analysis (Hb A1C)

- Slit lamp examination.

- Intraocular pressure (IOP) measurement

- Fundus examination.

- Fundus fluorescein angiography (FFA) to detect proliferative and non proliferative diabetes according to the leakage pattern.

- Optical Coherence Tomography (OCT) scan acquisition is grading parameters of diabetic macular edema

\subsection{Statistical Analysis}

Data were collected and entered to the computer using SPSS (Statistical Package for Social Science) program for statistical analysis (Chicago,Inc, Illinose, program for statistical analysis) version 18. Data were entered as numerical or categorical, as appropriate. Quantitative data were shown as mean \pm SD (min-max). Qualitative data were expressed as frequency and percent (\%). Chi- square test was used to measure association between qualitative variables. Independent sample t-test was done to compare the means of 2 sets of quantitative parametric data. Pearson correlation was done to assess the correlation between 2 sets of quantitative data. $\mathrm{P}$ (probability) value was considered to be of statistical significance if it is $\leq 0.05$

\section{Results}

In this study, $42.7 \%$ of the patients were males and $57.3 \%$ were females. The mean of the age was $41.49 \pm 6.14$ years. table (1).

The mean of the IOP was $17.28 \pm 1.05$. The mean of the BCVA was $0.36 \pm 0.2$. The means of central, retinal and cube thickness were $365.89 \pm 115.08$, $336.3 \pm 67.27$ and 9.38 \pm 1.98 ; respectively. table (2)

Table (1) The gender and age distribution in the studied group.

\begin{tabular}{llcc}
\hline & & \multicolumn{2}{c}{ Diabetic patients with macular oedema } \\
& & N=117 & $\%$ \\
\hline \multirow{2}{*}{ Gender } & Male & No & $42.7 \%$ \\
& Female & $\mathbf{5 0}$ & $\mathbf{5 7 . 3 \%}$ \\
Age (Years) & Mean \pm SD & $\mathbf{6 1 . 4 9 \pm 6 . 1 4}$ & \\
\hline
\end{tabular}

Table 2 The IOP, BCVA, and CMT, average retinal thickness and cube volume in the studied group

\begin{tabular}{lcc}
\hline & \multicolumn{2}{c}{ Diabetic patients with macular edema } \\
& Min- Max & Mean \pm SD \\
IOP $(\mathrm{mmHg})$ & $15-19$ & $17.28 \pm 1.05$ \\
BCVA & $0.05-0.67$ & $0.36 \pm 0.2$ \\
CMT $(\mu \mathrm{m})$ & $250-698$ & $365.89 \pm 115.08$ \\
Average retinal thickness $(\mu \mathrm{m})$ & $236-591.3$ & $336.3 \pm 67.27$ \\
Cube volume $(\mathrm{mm} 3)$ & $5.16-16.71$ & $9.38 \pm 1.98$ \\
\hline
\end{tabular}


There was significant negative correlation between the CMT and the BCVA $(\mathrm{P}<0.05)$. And significant positive correlation between the CMT and average retinal thickness and cube volume $(\mathrm{P}<0.05)$. table (3)

Table 4 shows that cystoid pattern of edema was more prevelant in patients with hard exudate, haemorrhage, absent foveal contour and disturbed ELM,RPE,PRL $(\mathrm{P}<0.05)$.

table 5 shows that patients with loss of foveal contour had significantly higher cystoid pattern of edema, lower BCVA and increased CMT than patients with preserved foveal contour $(\mathrm{P}<0.05)$.

Table (3) Correlation between the CMT and the patients' parameters.

\begin{tabular}{lcc}
\hline & r & CMT \\
\hline BCVA & -0.916 & P \\
age & -0.022 & $<0.0001$ \\
Duration of DM & 0.180 & 0.814 \\
HbA1c & 0.129 & 0.052 \\
IOP & -0.078 & 0.165 \\
Average retinal thickness & 0.679 & 0.400 \\
Cube volume & 0.733 & $<0.0001$ \\
\hline
\end{tabular}

Table (4) Comparison of the ophthalmological findings between cystoid and Sponge edema.

\begin{tabular}{|c|c|c|c|c|c|}
\hline & & \multicolumn{4}{|c|}{ Pattern of edema } \\
\hline & & Cystoid & Sponge & $\mathbf{X} 2$ & $\mathbf{P}$ \\
\hline & & $\mathrm{N}=52$ & $\mathrm{~N}=65$ & & \\
\hline \multirow[t]{2}{*}{ FFA } & PDR & $17(32.7 \%)$ & $16(24.6 \%)$ & 0.931 & 0.335 \\
\hline & NPDR & $35(67.3 \%)$ & $49(75.4 \%)$ & & \\
\hline \multirow[t]{2}{*}{ Hyperreflective foci } & yes & $36(69.2 \%)$ & $52(80.0 \%)$ & 1.797 & 0.180 \\
\hline & no & $16(30.8 \%)$ & $13(20.0 \%)$ & & \\
\hline \multirow[t]{2}{*}{ Hard exaudate } & yes & $32(61.5 \%)$ & $28(43.1 \%)$ & 3.941 & 0.047 \\
\hline & no & $20(38.5 \%)$ & $37(56.9 \%)$ & & \\
\hline \multirow[t]{2}{*}{ Hage } & yes & $37(71.2 \%)$ & $31(47.7 \%)$ & 6.533 & 0.011 \\
\hline & no & $15(28.8 \%)$ & $34(52.3 \%)$ & & \\
\hline \multirow[t]{2}{*}{ Subretinal fluid } & yes & $19(36.5 \%)$ & $12(18.5 \%)$ & 4.847 & 0.028 \\
\hline & no & $33(63.5 \%)$ & $53(81.5 \%)$ & & \\
\hline \multirow[t]{2}{*}{ foveal contour } & Preserved & $15(28.8 \%)$ & $55(84.6 \%)$ & $37.38^{\mathrm{a}}$ & $<0.0001$ \\
\hline & loss & $37(71.2 \%)$ & $10(15.4 \%)$ & & \\
\hline \multirow[t]{2}{*}{ ELM,RPE,PRL } & Intact & $11(21.2 \%)$ & $48(73.8 \%)$ & $32.08^{\mathrm{a}}$ & $<0.0001$ \\
\hline & Disturbed & $41(78.8 \%)$ & $17(26.2 \%)$ & & \\
\hline
\end{tabular}

Table (5) Comparison of the BCVA, Pattern of edema, types of DR and CMT between patients preserved and loss of foveal contour.

\begin{tabular}{llllll}
\hline & & $\begin{array}{l}\text { Foveal contour } \\
\text { Preserved } \\
\text { N=70 }\end{array}$ & $\begin{array}{l}\text { loss } \\
\mathbf{N}=\mathbf{4 7}\end{array}$ & X2 & P-value \\
Pattern of edema & $\begin{array}{l}\text { cystoid } \\
\text { Sponge }\end{array}$ & $55(78.6 \%)$ & $10(21.3 \%)$ & $37.385^{\mathrm{a}}$ & $<0.0001$ \\
& PDR & $18(25.7 \%)$ & $15(31.9 \%)$ & $0.534^{\mathrm{a}}$ & 0.465 \\
FFA & NPDR & $52(74.3 \%)$ & $32(68.1 \%)$ & & \\
BCVA & Mean \pm SD & $0.47 \pm 0.17$ & $0.198 \pm 0.104$ & $\mathrm{t}=9.836$ & $<0.0001$ \\
CMT $(\boldsymbol{\mu m})$ & Mean \pm SD & $297.9 \pm 53.18$ & $467.02 \pm 108.11$ & $\mathrm{t}=-11.22$ & $<0.0001$ \\
\hline
\end{tabular}




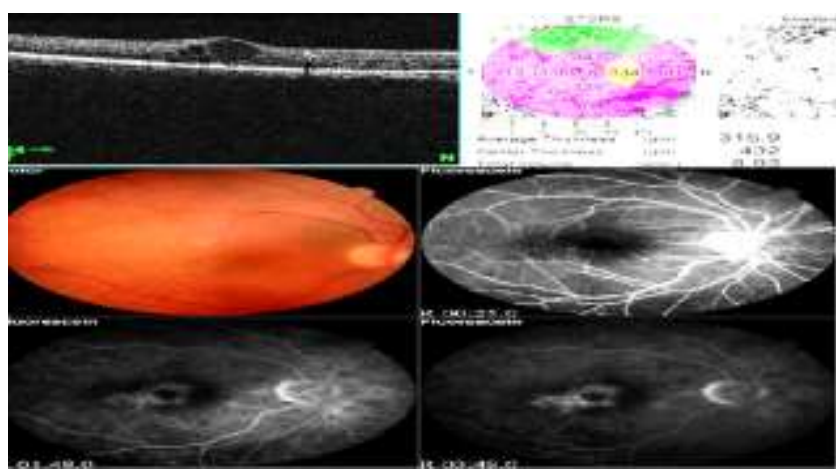

Fig. (1) Right eye showing cystoid macular edema with increased central thickness and BCVA 0.25 and loss of foveal contour.

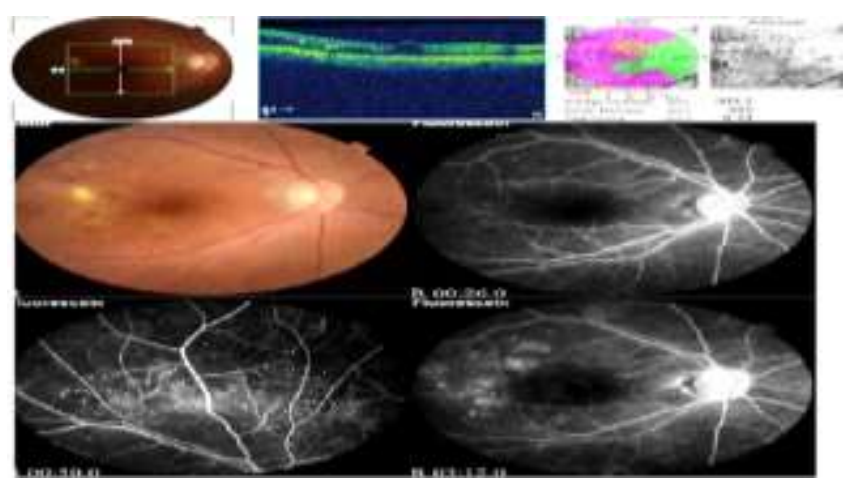

Fig. (2) Left eye of showing CME with BCVA 0.33

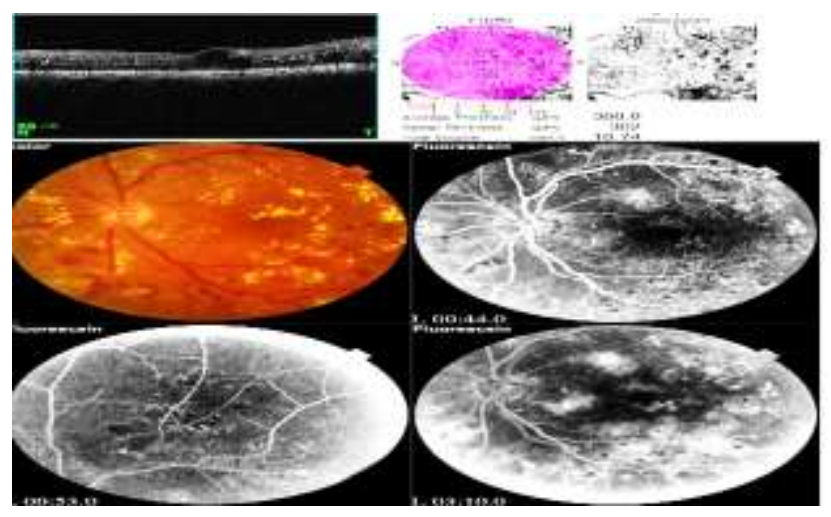

Fig. (3) Left eye showing,NPDR with hage,hard exudate,CME and BCVA 0.25.

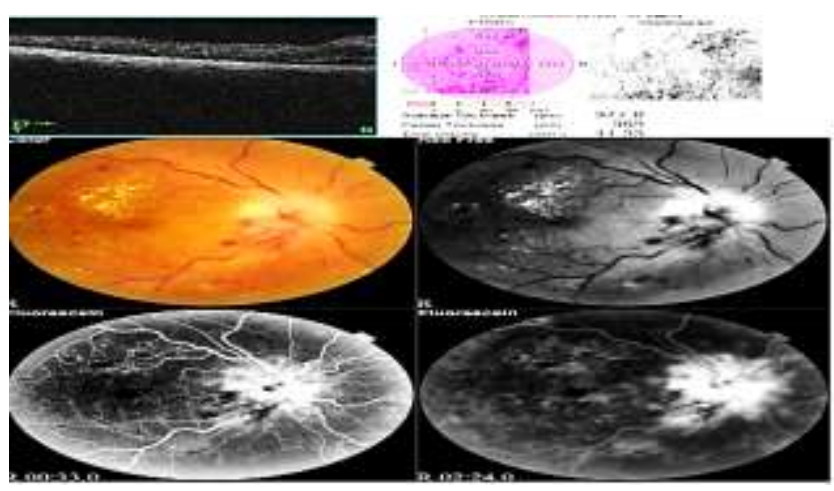

Fig. (4) Right eye showing PDR with hge,sponge macular edema and BCVA 0.25 


\section{Discussion}

In the current investigation, BCVA in patients with cystoid edoema was considerably lower than in patients with sponge edoema $(\mathrm{P}<0.05)$. In individuals with cystoid edoema, the central, retinal and cube volumes were considerably greater $(\mathrm{P}<0,05)$.

The existence of CMEs engaging the centre is thus the most critical OCT that affects vision.

These findings were similar with earlier research showing a slight connection between CMT and BCVA assessed by OCT.

Yamamoto et al.[9] reported that the fovea of cystoid edema's eyes was much thicker than the fovea of diffuse swelling of eyes. The mean retinal thickness and mean visual acuities likewise differed across groups based on morphological pattern in Kim et al.[10]; increased retinal thickness in all patterns was substantially connected with lower visual acuity.

Otani et al.[11] also found, in their study, that retinal thickening of central fovea and VA with best correction shows an intermediate negative correlation irrespective of the different tomography characteristics whereas Murakami et al.[12] found that the median MAR VA log for the CME type is much worse than for the SRD or diffuse type but found that parafoveal thickeni

Another study assessed the link between visual and microstructural alterations in DME's internal segment-outer (IS/OS) junction and external limiting membrane (ELM). [13] [13]

Uji et al. [14] reported in their research that the existence of hyperreflective fibres in the external retina was strongly linked to an ELM and IS/OS connection on SD-OCT images and a reduction of the $\mathrm{VA}$ in DME.

In summary, our research demonstrated that OCT reported three fundamental DME alterations, including sponge-like retinal edoema, CME and SRD. The worst visual result was for CME.

Chou et al. [15] investigation indicated an increased foveal thickness evaluated by OCT in diabetic eyes for individuals with HbalC of eight percent or more, and statistical link existed between HbalC and CMT. Do et al;[16] reveals that those with persistent CSME are at the time of their diagnosis statistically significantly higher than those with resolved CSMEs. These findings show that better glycemic controls may prevent chronic CSME development. Anand et al;[17] pointed out that a single measurement $(\mathrm{HbA} 1 \mathrm{c})$, in particular because of the long-lasting occurrence of persistent CSMEs, might not really represent glycemic management. They agreed that the acquisition of average HbA1c serial readings would give additional information for the research population and reinforce the connection between HbA1c levels and persistent or resolved CSMEs. Varma et al;[18] reported a higher burden of DME among non-Hispanic blacks, those with high HbA1c values, and those who had longer diabetes durations. Ozer et al;[19] concluded that there was no association between blood lipid levels and DME severity, although the duration of diabetes was a major determinant for macular edoema development. High values of HbA1c in all individuals emphasise the significance of intensive glycemic management in diabetic patients.

In our research we discovered no statistical link between $\mathrm{HbAlC}$ and $\mathrm{CMT}$, which was mostly attributable to $\mathrm{HbAlC}$ for all candidates. Also, the statistical link between DME and diabetes duration was minor, mainly because our candidates had a lengthy diabetes length.

\section{Conclusion}

The purpose of our study was to evaluate DME using (SD- OCT) and correlate the results with BCVA ,HbA 1c and the duration of diabetis in eyes with untreated DME. Cystoid macular edema and loss of foveal contour associated with the most worest visual acuity. No significant differences were detected between CMT and HbAlc. There was insignificant statistical correlation between CMT \& duration of diabetes.

\section{References}

[1] J.A.Wells Aflibercept, bevacizumab, or ranibizumab for diabetic macular edema: twoyear results from a comparative effectiveness randomized clinical trial," Ophthalmology, vol. 123, pp. 1351-1359, 2016.

[2] N. H. Arar . "Heritability of the severity of diabetic retinopathy: the FIND-Eye study," Invest. Ophthalmol. Vis. Sci., vol. 49, pp. 3839-3845, 2008.

[3] G.Coscas, J.Cunha-Vaz, and A. Loewenstein, Macular edema: a practical approach, vol. 47. Karger Medical and Scientific Publishers, vol. 1, pp. 65-67, 2010.

[4] B.S.Gerendas et al., "Predictive imaging biomarkers relevant for functional and anatomical outcomes during ranibizumab therapy of diabetic macular oedema," Br. J. Ophthalmol., vol. 102, pp. 195-203, 2018.

[5] K. A. Joltikov. "Multidimensional functional and structural evaluation reveals neuroretinal impairment in early diabetic retinopathy," Invest. Ophthalmol. Vis. Sci., vol. 58, no. 6, pp. BIO277-BIO290, 2017.

[6] H. J. Zambarakji, W. M. Amoaku, and S.A.Vernon, "Volumetric analysis of early macular edema with the Heidelberg Retina Tomograph in diabetic retinopathy," Ophthalmology, vol. 105, pp. 1051-1059, 1998.

[7] Ş. C. Özdek, M. A. Erdinç, G. Gürelik, B. Aydın, U. Bahçeci, and B. Hasanreisoğlu, "Optical coherence tomographic assessment of diabetic macular edema: comparison with fluorescein angiographic and clinical 
findings," Ophthalmologica, vol. 219, pp. 8692, 2005.

[8] P.K.Kaiser, C.D.Riemann, J.E.Sears, and H. Lewis, "Macular traction detachment and diabetic macular edema associated with posterior hyaloidal traction," Am. J. Ophthalmol., vol. 131, pp. 44-49, 2001.

[9] S.Yamamoto, T.Yamamoto, M.Hayashi, and S. Takeuchi, "Morphological and functional analyses of diabetic macular edema by optical coherence tomography and multifocal electroretinograms," Graefe's Arch. Clin. Exp. Ophthalmol., vol. 239, pp. 96-101, 2001.

[10] N.R.Kim, Y. J. Kim, H.S.Chin, and Y.S.Moon, "Optical coherence tomographic patterns in diabetic macular oedema: prediction of visual outcome after focal laser photocoagulation," Br. J. Ophthalmol., vol. 93, pp. 901-905, 2009.

[11] T. Otani, Y. Yamaguchi, and S. Kishi, "Correlation between visual acuity and foveal microstructural changes in diabetic macular edema," Retina, vol. 30, pp. 774-780, 2010.

[12] T. Murakami, K. Nishijima, A. Sakamoto, M. Ota, T. Horii, and N. Yoshimura, "Association of pathomorphology, photoreceptor status, and retinal thickness with visual acuity in diabetic retinopathy," Am. J. Ophthalmol., vol. 151, pp. 310-317, 2011.

[13] Y. Shen, K. Liu, and X. Xu, "Correlation between visual function and photoreceptor integrity in diabetic macular edema: spectraldomain optical coherence tomography," Curr. Eye Res., vol. 41, pp. 391-399, 2016.

[14] A. Uji . "Association between hyperreflective foci in the outer retina, status of photoreceptor layer, and visual acuity in diabetic macular edema," Am. J. Ophthalmol., vol. 153, no. 4, pp. 710-717, 2012.

[15] T. H.Chou, P.C.Wu, J.Z.C.Kuo, C. H. Lai, and C. N. Kuo, "Relationship of diabetic macular oedema with glycosylated haemoglobin," Eye, vol. 23, pp. 1360-1363, 2009.

[16] D. V Do, S. M. Shah, J. U. Sung, J. A. Haller, and Q. D. Nguyen, "Persistent diabetic macular edema is associated with elevated hemoglobin A1c," Am. J. Ophthalmol., vol. 139, pp. 620-623, 2005.

[17] A. Anand, S. Negi, and W. J. Kalk, "Persistent diabetic macular edema is associated with elevated hemoglobin A1c," Am. J. Ophthalmol., vol. 140, pp. 960-961, 2005.

[18] R. Varma et al., "Prevalence of and risk factors for diabetic macular edema in the United States," JAMA Ophthalmol., vol. 132, pp. 1334-1340, 2014.

[19] P. A. Ozer, N. Unlu, M. N. Demir, D. O. Hazirolan, M. A. Acar, and S. Duman, "Serum lipid profile in diabetic macular edema," J. Diabetes Complications, vol. 23, pp. 244-248, 2009. 